\section{Radiation-induced spinal cord hemorrhage (hematomyelia)}

\author{
Amit Agarwal, 1 Sangam Kanekar,2 \\ Krishnamurthy Thamburaj,1 \\ Kanupriya Vijay ${ }^{1}$
}

1Department of Radiology, Penn State

University Hershey, PA; 2Department of

Neurology and Radiology, University

Drive, Hershey, PA, USA

\begin{abstract}
Intraspinal hemorrhage is very rare and intramedullary hemorrhage, also called hematomyelia, is the rarest form of intraspinal hemorrhage, usually related to trauma. Spinal vascular malformations such intradural arteriovenous malformations are the most common cause of atraumatic hematomyelia. Other considerations include warfarin or heparin anticoagulation, bleeding disorders, spinal cord tumors. Radiation-induced hematomyelia of the cord is exceedingly rare with only one case in literature to date. We report the case of an 8 year old girl with Ewing's sarcoma of the thoracic vertebra, under radiation therapy, presenting with hematomyelia. We describe the clinical course, the findings on imaging studies and the available information in the literature. Recognition of the clinical pattern of spinal cord injury should lead clinicians to perform imaging studies to evaluate for compressive etiologies.
\end{abstract}

\section{Introduction}

Intramedullary spinal cord hemorrhage (hematomyelia) is an uncommon cause of myelopathy and can present in an acute, subacute, stepwise, or chronic fashion. ${ }^{1}$ There are very few reports of hematomyelia in the medical literature, most in relation to anticoagulant treatment. Among the predisposing factors, anticoagulation, trauma, arteriovenous malformations, tumors, and hemorrhagic diathesis can be included. ${ }^{2}$ We present the case of a child with Ewing's sarcoma of the thoracic spine developing hematomyelia as a delayed complication of radiation therapy. Radiation damage to the spinal cord is one of the most feared complications in the treatment of cancer with radiation therapy. It is widely accepted that the therapeutic index of radiotherapy limits the radiation dose near the spinal cord to such an extent that the control of many tumors is compromised. ${ }^{3}$ Radiation-induced hematomyelia of the cord is however, an exceedingly rare manifestation of radiation cord injury with only one case in literature to date.

\section{Case Report}

An 8-year old girl presented to our hospital with back pain for a couple weeks and had weakness in her lower extremities. Clinical examination revealed arflexia and loss of motor power in both her lower extremities. Magnetic resonance imaging (MRI) of the spine done subsequently revealed an epidural mass at the level of T7-T9 which was circumferential and was compressing her spinal cord with marrow signal changes within the T8 vertebral body (Figure 1). She was immediately started on oral Dexamethasone and underwent an emergent debulking of the mass along with decompressive thoracic laminectomies. Since the mass was circumferential, it was difficult to excise the mass in total. The final pathology was consistent with Ewing sarcoma. She did well post-operatively and regained her lower extremity functions and strength over the next few weeks. She had a post-op MRI which showed expected postsurgical changes and residual ventral epidural enhancing mass at T7-T9 level. There was no metastatic disease. She was started on chemotherapy as per protocol and also underwent proton beam radiotherapy. Proton therapy dose of 46 Gray (Gy), given in 1.8 Gy daily fractions, was delivered to the clinical target volume. Multiple follow-up MRI scans showed complete resolution of the residual epidural mass and expected post-treatment changes, including fatty marrow replacement and mild cord atrophy (Figure 2). Around seven months after the last administered radiotherapy dose, the patient developed new sensory-motor symptoms in the lower limbs including mild parasthesias and loss of motor power. MRI of the thoracic spine done soon after showed a large hematomyelia within the thoracic cord spanning from T5-T11 level (Figure 3). This was seen as a long segment T1 hyperintense, T2 hypointense area within the central cord with blood-fluid level at the cephalic and caudal end. There was moderate expansion of the cord, especially evident when compared to the prior scans. There was no pathologic enhancement to suggest any intramedullary lesion. The patient was monitored for few days with no progression of symptoms. Patient was discharged and the intramedullary hematoma was left to spontaneous resolution. Follow-up MR imaging after 6 weeks showed decrease in intramedullary hemorrhage, expected evolution of blood products and resolution of cord swelling. Again, no intramedullary vascular malformation or tumor was identified on the follow-up MRI.
Correspondence: Amit Agarwal, Department of Radiology, Penn State University Hershey Medical Center, 500 University Dr Hershey, 17033 PA, USA.

Tel.: +1.717.531.6737 - Fax: +1.717.531.0006

E-mail: aagarwal1@hmc.psu.edu;

amitmamc@gmail.com.

Key words: hematomyelia, intramedullary, spinal cord. radiation.

Contributions: the authors contributed equally.

Conflict of interests: the authors declare no potential conflict of interests.

Received for publication: 15 July 2014. Revision received: 15 September 2014.

Accepted for publication: 26 September 2014.

This work is licensed under a Creative Commons Attribution NonCommercial 3.0 License (CC BYNC 3.0).

(C) Copyright A. Agarwal et al., 2014

Licensee PAGEPress, Italy

Neurology International 2014; 6:5553

doi:10.4081/ni.2014.5553

\section{Discussion}

Intraspinal hemorrhage is much less common than intracranial hemorrhage and can be epidural, subdural, subarachnoid, or intramedullary with devastating consequences. Intramedullary hemorrhage, also known as hematomyelia, is the rarest form of intraspinal hemorrhage. ${ }^{4}$ Since the first report of spontaneous hematomyelia by Richardson, 5 few other case reports of hematomyelia have been described, most in relation to anticoagulant treatment. Among the predisposing factors, anticoagulation, trauma, arteriovenous malformations, tumors, and hemorrhagic diathesis can be included. The most frequent localization in children is $\mathrm{C} 5$ - $\mathrm{T} 1$, different from adults, more frequently found at a low cervical and thoracolumbar level.6,7

Hematomyelia usually presents with an acute onset and rapid deterioration in neurologic status and usually leads to an acute spinal cord syndrome. Prompt diagnosis of hematomyelia first requires recognition of a myelopathy syndrome (transverse, central, anterior, posterior, or hemi-cord) often accompanied by sudden, severe back or neck pain and sometimes radicular pain. Besides the common acute type of hematomyelia, there is documentation of step-wise or chronic progressive patterns of hematomyelia. ${ }^{8}$ The duration of the symptoms from onset to admission can range from 2 months to 6 years in chronic hematomyelia, but the symptoms usually progress within 5 months in the majority of the 
cases. $^{9}$

Radiation damage to the spinal cord is one of the most feared complications in the treatment of cancer with radiation therapy (RT). Its medical and legal consequences are such that radiation oncologists often compromise the treatment of the malignancy to ensure that the spinal cord dose remains at a safe level. ${ }^{10}$ Portions of the spinal cord are often included in RT fields for treatment of malignancies involving the neck, thorax, abdomen, and pelvis. In addition, metastatic disease to the bony spine, often requiring $\mathrm{RT}$, is encountered in $40 \%$ of all cancer patients. 11 The upper limit for a safe dose is generally defined as a total dose of 55-60 Gy delivered to a restricted field whenever possible, in 1.8-2.0 Gy daily fractions. However, despite these measures, the unpredictability of individual sensitivities to irradiation remains an issue. There are many cases of radiation myelitis reported in literature; however radiation-induced hematomyelia is exceedingly rare. Moreover, the pathogenesis of myelopathy after radiation is likely to be multifactorial, with no single target cell and no single pathway. Definite pathogen- ic relationship between radiation and spinal cord hemorrhage has not yet been established. Injury is likely mediated by damage to both white matter tracts and the local vasculature. This assertion is supported by the pathological observation of demyelination, necrosis, increased vascularity, telangiectasis, vasculitis, fibrin exudation, thrombosis, and edema in cases of spinal cord injury from radiation. ${ }^{3}$

We could find only one reported case of radiation-induced hematomyelia to date. This was reported by Purroy et al.12 in a 49 year old with breast malignancy. This patient had received multiple rounds of radiation and presented with acute intramedullary hemorrhage. No vascular malformation, trauma or coagulation disorder was identified as the underlying cause. Neurological examination was compatible with a Brown-Séquard syndrome. There was a motor deficit with exaltation of deep muscle reflexes, hypoesthesia with decreased vibratory sensation and no sphincter control. MR imaging revealed long segment T2 hypointense lesion within the thoracic cord with T1 hyper intensity. Imaging findings were very similar to those observed in our patient. As in our case, clinical symptoms were predominantly sensory-motor in nature. MRI examination is the best diagnostic tool for hematomyelia, although other common diagnostic tools are used: conventional myelography or CT myelogram. MRI is the method of choice because it depicts very well the hemorrhage and the possible cause of bleeding. MRI shows an intramedullary lesion with signal characteristics of hemorrhage of variable age on both the $\mathrm{T} 1$ and $\mathrm{T} 2$ weighted images, without enhancement. 12 MRI displays excellent contrast in the nervous tissue in the chronic stage of intraparenchymal hemorrhage, whereas the diagnostic ability of CT decreases because the chronic hematoma becomes isodense. With the increasing usage of MRI for clinical diagnosis, we may assume that there will be an increasing number of cases of chronic hematomyelia. Because the clinical course of chronic progressive hematomyelia resembles that of spinal tumors or other spinal progressive disease, MRI is essential for confirmation of the diagnosis. ${ }^{9}$ The role of angiography has been debated. Kaaravelis et al. indi-

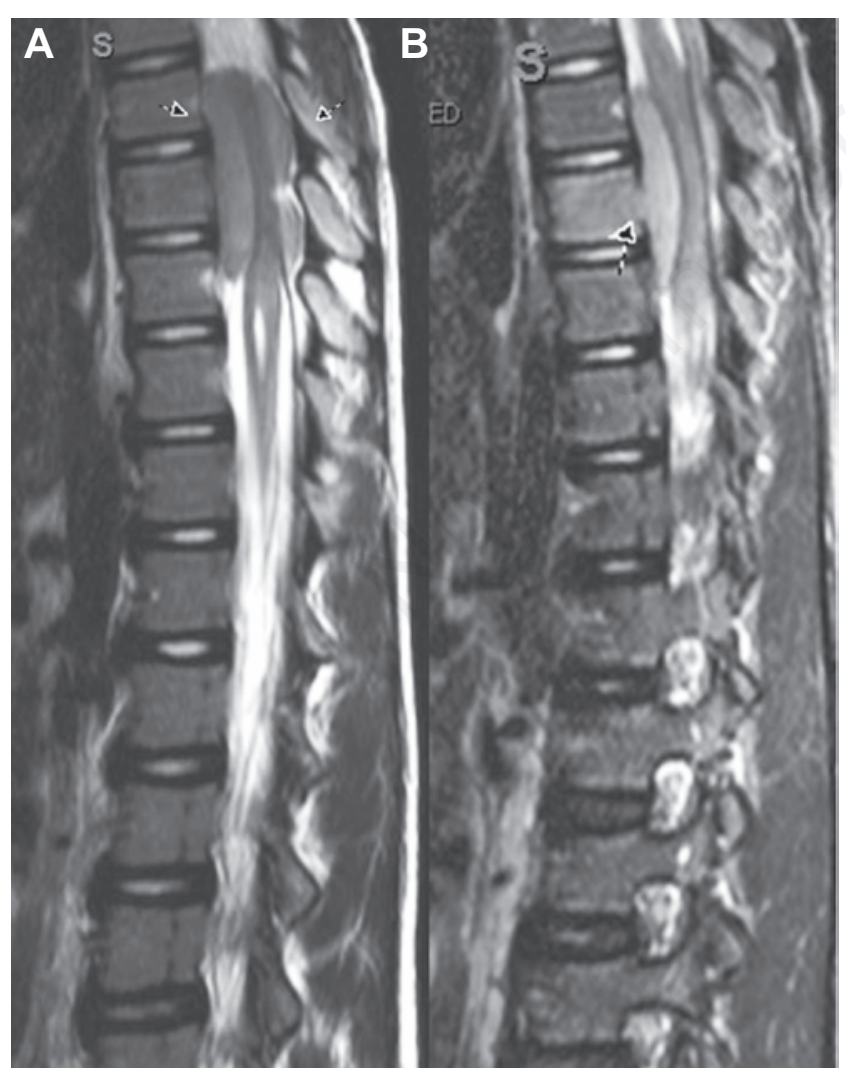

Figure 1. Sagittal T2-weighted magnetic resonance images $(A, B)$ through the mid thoracic spine shows a homogenous circumferential epidural mass compressing the spinal cord. The second image (B; arrow) shows posterior cortical disruption of the T8 body with marrow signal changes. The final pathology was consistent with Ewing sarcoma.

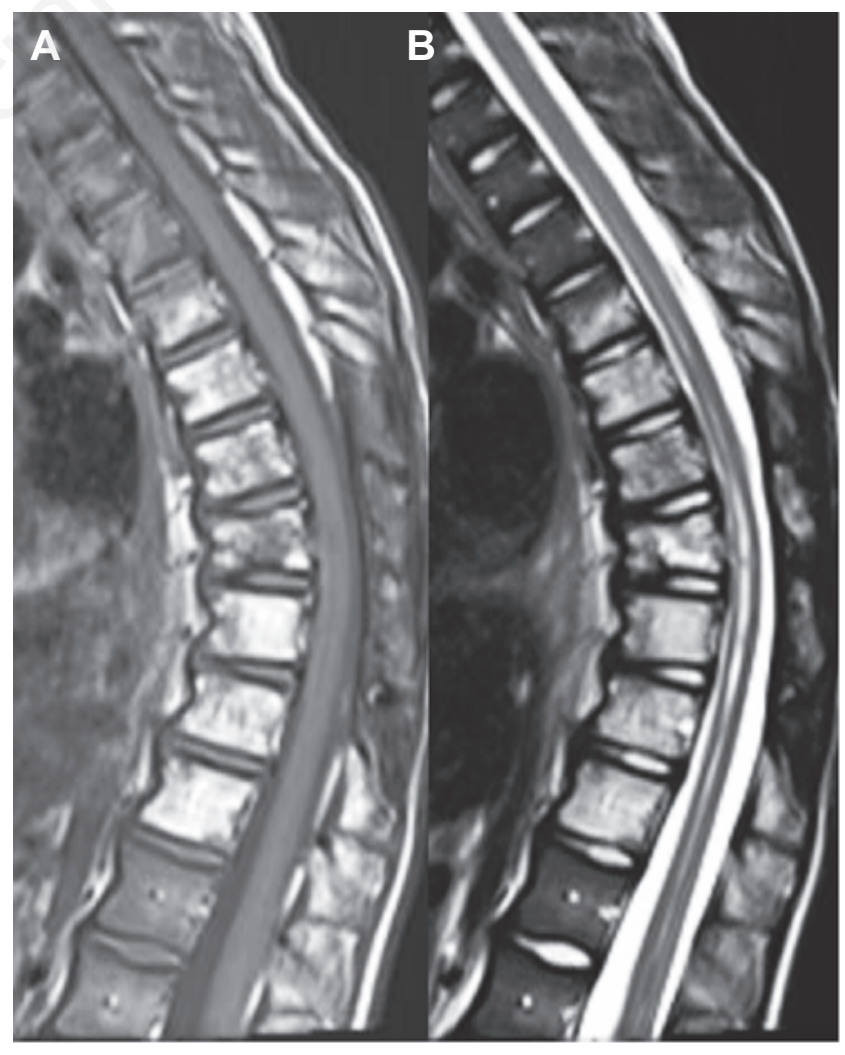

Figure 2. Sagittal T1 (A) and T2 (B) weighted images after 1 year of surgery shows fatty marrow changes involving multiple thoracic vertebrae. Post-surgical changes of decompressive laminectomies are also seen. There is mild atrophy of the mid thoracic spinal cord with prominent central canal, best seen on the T2 weighted images. No residual or recurrent tumor seen. 
cated that spinal angiography was not necessary, if MRI was diagnostic and did not show any abnormal vessels. 12 Treatment of cases with acute onset it usually an emergency laminectomy with evacuation of the hematoma. Treatment of the underlying pathological condition in the case of an associated lesion such as tumor, aneurysm, vascular malformation, and decompression of the cord seems to offer the best chance of minimizing spinal cord damage and preventing permanent neurological sequelae. 13
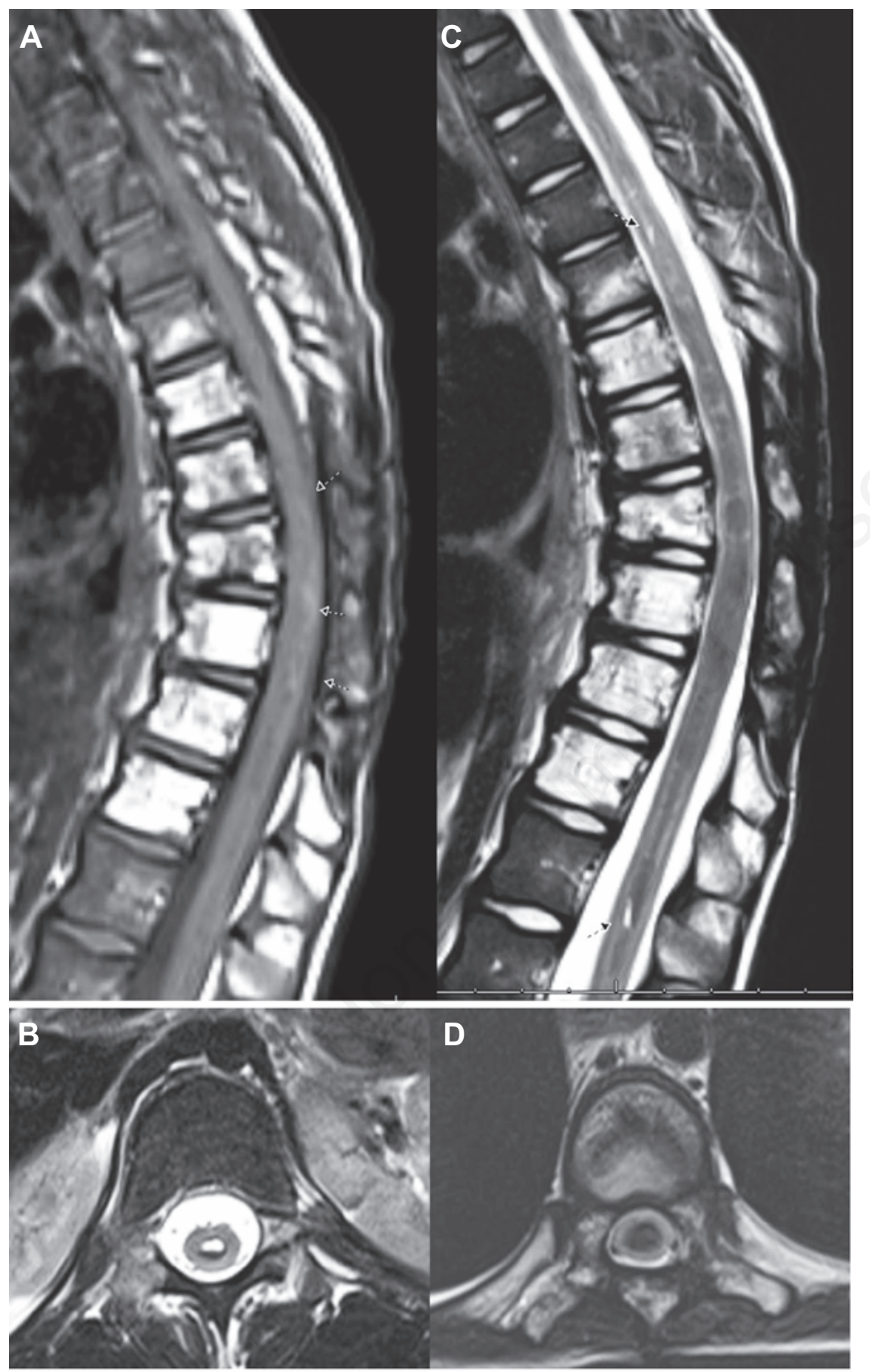

Figure 3. Sagittal and axial images from MRI scan seven months after the last administered radiotherapy dose. Sagittal T1-weighted images shows the heterogeneously T1 hyperintense areas within the thoracic cord ( $\mathrm{A}$; arrows) with corresponding low-signal on the T2 images. There is moderate expansion of the cord, especially evident on comparison with the prior scan. Fluid-blood level is noted at the caudal and cephalic end on the T2 sagittal image (B; arrows), also nicely seen on the T2 axial image (C). Signal dropout on the axial gradient image (D) confirms intramedullary hemorrhage.

\section{Conclusions}

In conclusion, spinal cord hematoma or hematomyelia is an infrequently encountered condition that is the result of several unusual disease processes. The causes of spontaneous, nontraumatic spinal cord hematoma include vascular malformations of the spinal cord (the most common), clotting disorders and spinal cord tumors. Radiation is an exceedingly rare cause of hematomyelia which can have an acute presentation with a Brown-Sequard type of syndrome or can present in a step-wise or chronic fashion. Clinical suspicion, an opportune diagnosis using MRI, and a timely intervention are essential in attaining a better neurological prognosis

\section{References}

1. Leep Hunderfund AN, Wijdicks EF. Intramedullary spinal cord hemorrhage (hematomyelia). Rev Neurol Dis 2009;6:E54-61.

2. Pullakart VA, Kalapura T, Pincus M, Baskharoun R. Intraspinal hemorrhage complicating oral anticoagulant therapy. Arch Intern Med 2000;160:237-40.

3. Gibbs IC, Patil C, Gerszten PC, et al. Delayed radiation-induced myelopathy after spinal radiosurgery. Neurosurgery 2009;64:A67-72.

4. Irazoque-Palazuelos F, Sosa-Espinosa PV, Andrade-Ortega L. Hematomyelia in systemic lupus erythematosus and secondary antiphospholipid syndrome: case report. Reumatol Clin 2008;4:34-6.

5. Richardson JC. Spontaneous hematomyelia: a short review and a case report illustrating intramedullary angioma and syphilis of the spinal cord as possible causes. Brain 1938;61:17-36.

6. Chen CJ, Hsu WC. Experience in the surgical management of spontaneous spinal epidural hematoma. J Formos Med Assoc 1997;96:283-7.

7. Patel H, Boaz JC, Philips JP. Spontaneous spinal epidural hematoma in children. Pediatr Neurol 1998;19:302-7.

8. Barnwell SL, Dowd CF, Davis RL, et al. Cryptic vascular malformations of the spinal cord: diagnosis by magnetic resonance imaging and outcome of surgery. $\mathrm{J}$ Neurosurg 1990;72:403-7.

9. Matsumura A1, Ayuzawa S, Doi M, et al. Chronic progressive hematomyelia: case reports and review of the literature. Surg Neurol 1999;51:559-63.

10. Marcus RB Jr, Million RR. The incidence of myelitis after irradiation of the cervical spinal cord. Int J Radiat Oncol Biol Phys 
1990;19:3-8.

11. Klimo P Jr., Thompson CJ, Kestle JR, et al. A meta-analysis of surgery versus conventional radiotherapy for the treatment of metastatic spinal epidural disease. Neuro Oncol 2005;7:64-76.

12. Purroy F, Arenillas JF, Rovira A, et al.
[Hematomyelia as late side effect of radiation]. Med Clin (Barc) 2002;119:799.

[Article in Spanish].

13. Kaaravelis A, Foroglou G, Petsanas A, Zarapoukas T. Spinal cord dysfunction caused by non-traumatic hematomyelia. Spinal Cord 1996;34:268-71.
14. Pullarkat VA, Kalapura T, Pincus M, et al. Intraspinal hemorrhage complicating oral anticoagulant therapy: an unusual case of cervical hematomyelia and a review of the literature. Arch Intern Med 2000;160:23740. 